\title{
Posterior-Chamber Phakic Intraocular Lens Implantation in Patients over 40 Years of Age
}

\author{
Pedro Tañá-Rivero, ${ }^{1}$ Francisco Pastor-Pascual, ${ }^{1}$ Marceliano Crespo, ${ }^{1}$ \\ José L. Rodríguez-Prats, ${ }^{1}$ José J. Muñoz-Tomás, ${ }^{1}$ and Robert Montés-Micó $\mathbb{D}^{1,2}$ \\ ${ }^{1}$ Oftalvist Clinic, Spain \\ ${ }^{2}$ Optics and Optometry \& Vision Sciences Dept, University of Valencia, Valencia, Spain \\ Correspondence should be addressed to Robert Montés-Micó; robert.montes@uv.es
}

Received 21 April 2020; Accepted 9 June 2020; Published 29 June 2020

Academic Editor: Inés Contreras

Copyright (c) 2020 Pedro Tañá-Rivero et al. This is an open access article distributed under the Creative Commons Attribution License, which permits unrestricted use, distribution, and reproduction in any medium, provided the original work is properly cited.

\begin{abstract}
Purpose. To assess the efficacy, safety, and predictability of the Visian Implantable Collamer Lens (ICL) model having a central port in patients over 40 years of age. Methods. This study included 33 eyes from 21 patients who underwent V4c ICL implantation for the correction of myopia and myopic astigmatism. We assessed uncorrected (UDVA) and corrected (CDVA) distance visual acuity, refraction, intraocular pressure (IOP), endothelial cell density (ECD), vault, and adverse events occurring over a 1-year period. Results. Mean age of the patients at the time of implantation was $43.52 \pm 4.49$ years (range: 40 to 56 years). Efficacy and safety indexes were 1 and 1.09, respectively. Surgical outcomes for CDVA were as follows: no eye lost any lines, 19 eyes (57.58\%) showed no CDVA changes, 7 eyes (21.21\%) gained 1 line, 4 eyes (12.12\%) gained 2 lines, and 3 eyes (9.09\%) gained $\geq 3$ lines. Mean postoperative spherical equivalent (SE) was $-0.09 \pm 0.47 \mathrm{D}$. A total of 29 eyes $(87.8 \%)$ were within $\pm 0.50 \mathrm{D}$ and 31 eyes $(93.9 \%)$ were within $\pm 1.00 \mathrm{D}$ of the desired SE. At 1 -year, mean IOP was $15.27 \pm 3.03 \mathrm{mmHg}$ (range: 9 to $20 \mathrm{mmHg}, p=0.12$ pre vs. post) and mean ECD was $2516 \pm 234$ cells $/ \mathrm{mm}^{2}$ ( $p=0.29$ pre vs. post). Mean postoperative vault was $320 \pm 136 \mu \mathrm{m}$, with $201-300 \mu \mathrm{m}$ being the most prevalent vault range for 9 eyes $(31.03 \%)$. None of the eyes showed a vault $>701 \mu \mathrm{m}$. There were neither intraoperative nor postoperative complications; in fact, all ICL implantation procedures were uneventful. Conclusions. Our study's findings support the use of this lens in patients over 40 years of age. A long follow-up period is advisable to monitor ICL position relative to the crystalline lens.
\end{abstract}

\section{Introduction}

The use of phakic intraocular lenses is an approach that is frequently used to correct different amounts of refractive errors. However, it is highly myopic patients-with or without astigmatism - who have especially benefited from the good visual and refractive outcomes that these intraocular lenses provide. One of the most popular phakic lenses is the posterior-chamber phakic Visian Implantable Collamer Lens (ICL) (Staar Surgical AG, Nidau, Switzerland), whose latest model (Visian V4c) incorporates a $0.36 \mathrm{~mm}$ central port (i.e., a hole) (KS-Aquaport). This opening allows the physiological flow of aqueous, thus making Nd:YAG peripheral iridotomies or intraoperative iridectomies unnecessary. Recent reviews and meta-analysis have confirmed its safety and effectiveness in moderately and highly myopic eyes throughout short, medium, and long follow-up periods $[1,2]$. With these outcomes, and bearing in mind that no vision-threatening complications occurred, the use of this IOL is recommended for the correction of a wide range of myopic errors.

However, few studies have included in their samples patients older than 40 years of age, and when they did so, the data analysis encompassed the whole sample, thus including patients of different ages (usually cohorts between 20 and 45 years). Consequently, specific studies are needed where this lens' performance is assessed as a function of the patient's age. Aging and, more specifically, the age-related changes 
that happen in the crystalline lens (i.e., increase in thickness) is an important factor to be considered when ICLs are implanted in people belonging to this age group. Two recent publications by the same group $[3,4]$ showed the outcomes for early presbyopic patients (aged 40 to 53; 6 months of follow-up period) but with a slight intentional postoperative undercorrection $[3,4]$. They concluded that is a viable surgical presbyopic treatment for this type of patients. Nonetheless, we believe that the use of these lenses in patients over 40 requires a more specific analysis so as to fully understand their clinical performance. Hence, the objective of this study was to assess the visual and refractive outcomes of the V4c Visian ICL model for the correction of myopia and astigmatism when implanted in patients over 40 years of age.

\section{Material and Methods}

2.1. Patients. A total of 33 eyes from 21 patients $(6$ males and 15 females) aged 40 to 56 were consecutively recruited for this prospective study. These patients underwent the implantation of the Visian V4c ICL to have their myopia and their myopic astigmatism corrected at the Oftalvist Clinic. The procedures took place between March 2017 and February 2019. The inclusion criteria were age $\geq 40$ years, and myopic and astigmatic refractive error to be corrected with the V4c ICL. The exclusion criteria were endothelial cell density (ECD) below 2000 cell $/ \mathrm{mm}^{2}$, anterior chamber depth $(\mathrm{ACD})<2.8 \mathrm{~mm}$, cataract, and a history of glaucoma or retinal detachment, neuro-ophthalmic diseases, macular degeneration, retinopathy, or any inflammatory disease. The study adhered to the tenets of the Declaration of Helsinki and was approved by the Institutional Review Board.

\subsection{Preoperative Examination. The preoperative examina-} tion included uncorrected distance visual acuity (UDVA), corrected distance visual acuity (CDVA), manifest refraction, slit-lamp, and a fundus examination carried out with ophthalmoscopy and using the Cirrus HD-OCT 5000 (Carl Zeiss Meditec AG, Jena, Germany). Moreover, Goldmann applanation tonometry was used to measure intraocular pressure (IOP) and ECD was measured with specular microscopy (SP-1P, Topcon, Tokyo, Japan). Lastly, for the anterior-segment analysis, the ANTERION Swept-Source Optical Coherence Tomography platform (SS-OCT, Heidelberg Engineering, Inc, Heidelberg, Germany) was used to measure ACD, white-to-white (WTW) distance, and angleto-angle (ATA) distance and for keratometry.

2.3. Phakic Intraocular Lens Selection. All eyes included in this study had either myopic or toric V4c ICLs implanted. ICL power calculation was carried out using a modified vertex formula according to the manufacturer's instructions. All eyes were targeted for emmetropia. ICL size calculation was based on the horizontal WTW, ACD, and ATA distances that had been measured with the ANTERION SSOCT. Topical antibiotic agents were used for 2 days before surgery. On the day of surgery, patients were administered
TABle 1: Preoperative patient demographics and ICL characteristics.

\begin{tabular}{lcc}
\hline & Mean \pm SD & Range [min, max] \\
\hline Age (years) & $43.52 \pm 4.49$ & {$[40,56]$} \\
Refraction sphere (D) & $-8.18 \pm 2.66$ & {$[-13.5,-2]$} \\
Refraction cylinder (D) & $-1.55 \pm 1.18$ & {$[-4.0,0]$} \\
Spherical equivalent (D) & $-8.46 \pm 3.31$ & {$[-14.88,-1.88]$} \\
Corneal thickness $(\mu \mathrm{m})$ & $535.18 \pm 36.66$ & {$[452,580]$} \\
CDVA (decimal) & $0.88 \pm 0.18$ & {$[0.40,1.00]$} \\
ACD $(\mathrm{mm})$ & $3.07 \pm 0.26$ & {$[2.67,3.59]$} \\
WTW $(\mathrm{mm})$ & $12.16 \pm 0.41$ & {$[11.30,12.70]$} \\
ATA $(\mathrm{mm})$ & $12.07 \pm 0.39$ & {$[11.50,12.79]$} \\
ECD (cells/mm2) & $2568 \pm 332$ & {$[2004,3297]$} \\
IOP (mmHg) & $16.23 \pm 3.61$ & {$[10,24]$} \\
ICL sphere (D) & $-9.52 \pm 2.65$ & {$[-15.0,-1.0]$} \\
ICL cylinder (D) & $2.65 \pm 1.23$ & {$[1,4.5]$} \\
ICL size $(\mathrm{mm})$ & $12.96 \pm 0.35$ & {$[12.10,13.20]$} \\
\hline
\end{tabular}

ICL: implantable Collamer lens; SD: standard deviation; D: diopters; CDVA: corrected-distance visual acuity; ACD: anterior chamber depth; WTW: white-to-white; ATA: angle-to-angle; ECD: endothelial cell density; IOP: intraocular pressure.

tropicamide eyedrops. Following topical anaesthesia, the ICL was introduced into the anterior chamber through a $2.75 \mathrm{~mm}$ temporal corneal incision or on the steepest meridian for astigmatism $\angle 1.00 \mathrm{D}$ using a STAAR injector and cartridge (Staar Surgical AG, Nidau, Switzerland) once the chamber had been filled with a viscosurgical device (Provisc, Alcon Laboratories Inc, Fort Worth, TX, USA). This viscosurgical device was completely removed at the end of the procedure. Once surgery was completed, dexamethasone and antibiotic medications were topically administered 5 times per day for 3 days, 3 times per day for 2 weeks, with the dose being reduced gradually thereafter. Follow-up visits were scheduled at 1 day, 5 days, and 1, 4, and 12 months postoperatively.

2.4. Postoperative Examination. Postoperative data were collected at the follow-up visits occurring at 1 day, 5 days, and 1, 4, and 12 months. For the purpose of this study, UDVA, CDVA, manifest refraction, IOP, and ECD values obtained at the 1-year follow-up visit were compared with preoperative values. Moreover, central vault height (i.e., the distance from the ICL's posterior surface to the crystalline lens' anterior surface) was also measured with the ANTERION SS-OCT as part of the postoperative examination. The same examiner performed all examinations.

2.5. Outcomes Analysis. Preoperative and 1-year postoperative visual acuity values - both UDVA and CDVA-were used to assess the efficacy and the safety of the procedure. As for predictability, the endpoint was the attempted-versusachieved manifest refraction. For this purpose, manifest refraction in conventional script notation ( $\mathrm{S}$ [sphere], $\mathrm{C}$ [cylinder], a [axis]) was converted into power vector coordinates: $\mathrm{SE}=S+\mathrm{C} / 2, J_{0}=(-\mathrm{C} / 2) X \cos (2 \alpha)$, and $J_{45}=(-\mathrm{C} /$ 2) $X \sin (2 \alpha)$, where $S E$ is the spherical equivalent and $J_{0}$ and $J_{45}$ are the two Jackson crossed-cylinders equivalent to the 


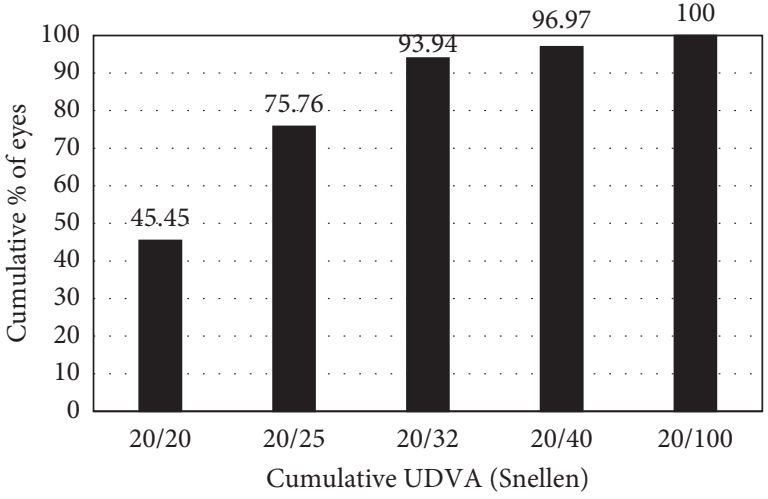

(a)

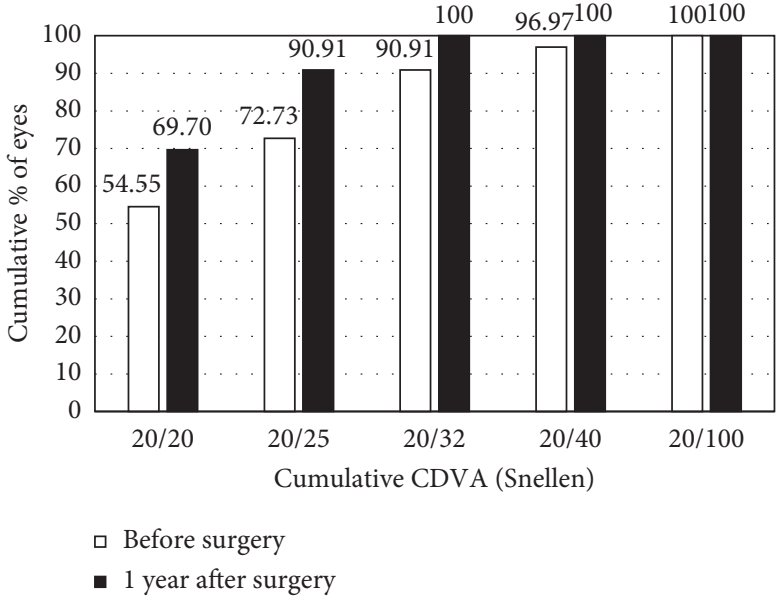

(b)

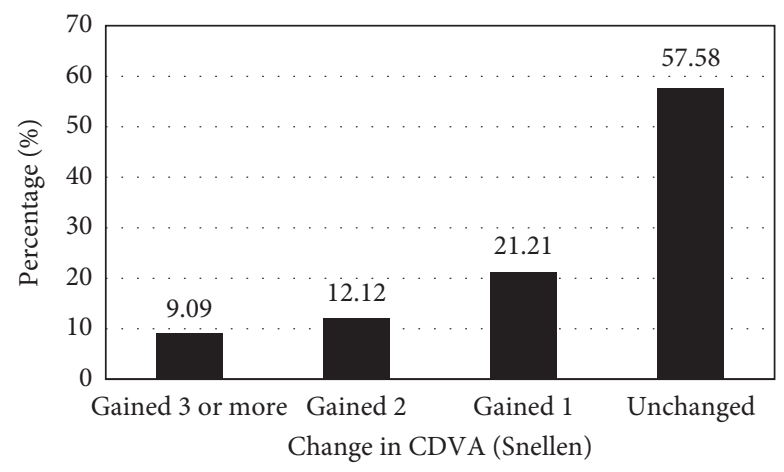

(c)

FIgURe 1: Cumulative uncorrected distance visual acuity (UDVA), at 1 year after surgery (a), cumulative corrected distance visual acuity (CDVA) before and at 1 year after surgery (b), and postoperative changes in corrected distance visual acuity (CDVA) at 1 year after surgery (c).

conventional cylinder. The efficacy index (defined as the ratio between postoperative UDVA and preoperative CDVA) and the safety index (defined as the ratio between postoperative CDVA and preoperative CDVA) were calculated based on Snellen decimal visual acuity values. Data analysis was performed using Excel (2016 version, Microsoft Corporation, Redmond, WA, USA). The Wilcoxon signedrank test was used to perform the preoperative-vs-postoperative data comparison. The results shown represent mean \pm standard deviation (SD) values; a $p$ value below 0.05 was considered to be statistically significant.

\section{Results}

The study comprised 33 eyes from 21 patients. Mean age of the 6 men $(28.6 \%)$ and 14 women (71.4\%) was $43.52 \pm 4.49$ years (range from 40 to 56 years). Mean preoperative SE refractive error was $-8.46 \pm 3.31 \mathrm{D}$ (range: -14.88 to $-1.88 \mathrm{D}$ ). Table 1 shows a summary of preoperative patient demographic data and implanted ICL characteristics. A toric ICL was implanted in 10 eyes (30.3\%); those were the ones requiring astigmatism correction of $1.5 \mathrm{D}$ or more. Regarding ICL diameter, 2 eyes were implanted with the 12.1 model, 9 eyes with the 12.6 model, and 22 eyes with the 13.2 model. Visual acuity outcomes, refractive error with SE and astigmatism vector analysis, IOP, ECD, vault, and adverse events were evaluated. The standard graphs for reporting refractive and visual acuity outcomes were constructed for each parameter analysed.

3.1. Visual Acuity Outcomes: Safety and Efficacy. At 1 year, mean postoperative Snellen decimal UDVA and CDVA were $0.88 \pm 0.16$ and $0.96 \pm 0.09$, respectively. Figure 1 shows the cumulative UDVA at 1 year after surgery (A, top), and CDVA before and 1 year after surgery $(B$, middle). As can be seen in those graphs, at 1 year a total of 25 eyes $(75.76 \%)$ and 30 eyes (90.91\%) had achieved an UDVA and CDVA of 20/ 25 or better, respectively. Figure 1 (C, bottom) illustrates the postoperative changes in CDVA at 1 year (relative to preoperative values). None of the eyes lost any lines, 19 eyes (57.58\%) maintained their preoperative CDVA, 7 eyes (21.21\%) gained 1 line, 4 eyes (12.12\%) gained 2 lines, and 3 eyes $(9.09 \%)$ gained 3 or more lines of CDVA. The resulting efficacy and safety indexes were 1 and 1.09, respectively.

3.2. Predictability. Figure 2(a) illustrates postoperative SE accuracy measured 1 year after surgery; in particular, it shows that for 22 eyes (66.7\%) SE refraction was within the 


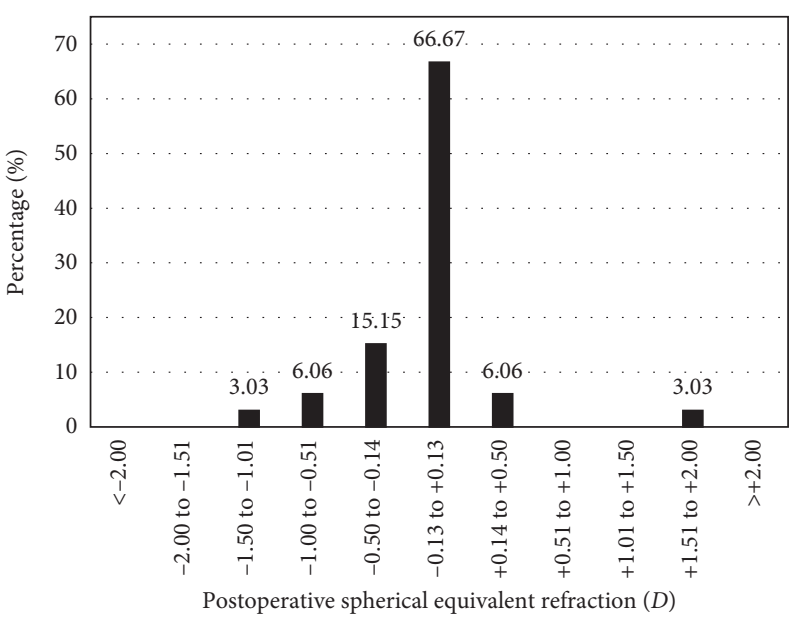

(a)

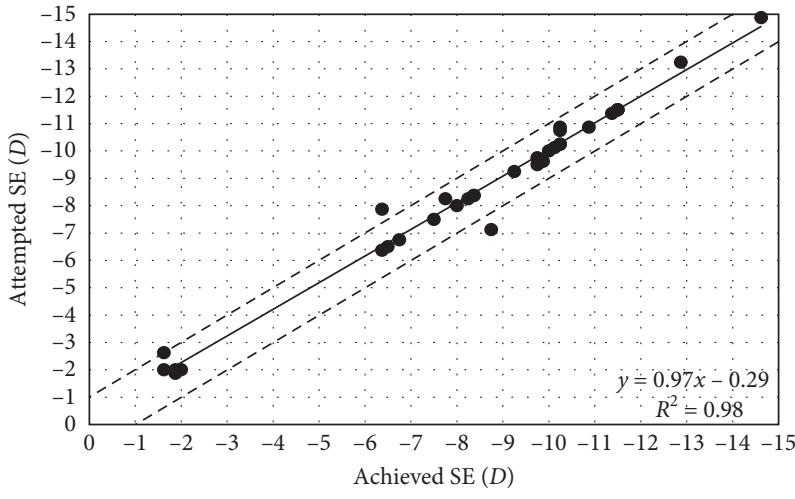

(b)

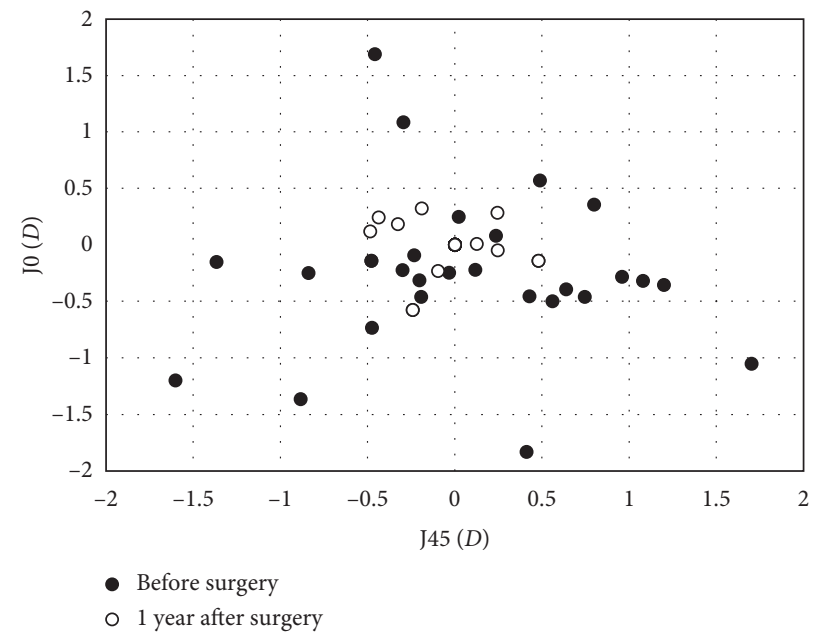

(c)

Figure 2: Postoperative spherical equivalent accuracy (a), attempted-versus-achieved spherical equivalent (b), and scatterplot of preoperative and 1-year postoperative astigmatic vectors (c). Solid line in figure B represents the best-fit line (regression equation and $\mathrm{R}$ value was included) and dotted lines $\pm 1.00 \mathrm{D}$. In the third figure, the more central location of postoperative data around 0 represents a reduction in astigmatism after lens implantation $\left(J 0=\right.$ Jackson cross-cylinder, axes at $180^{\circ}$ and $90^{\circ} ; J 45=$ Jackson cross-cylinder, axes at $45^{\circ}$ and $\left.135^{\circ}\right)$.

$\pm 0.13 \mathrm{D}$ range (emmetropia). Mean postoperative SE was $-0.09 \pm 0.47$ D. Figure $2(\mathrm{~b})$ is a scatterplot of the attemptedversus-achieved SE at 1 year after surgery. 29 eyes $(87.8 \%)$ were within $\pm 0.50 \mathrm{D}$ and 31 eyes $(93.9 \%)$ were within \pm 1.00 D. Figure 2(c) shows the astigmatic component of the power vector as represented by a 2-dimensional vector (J0, J45). In this graph $(0,0)$ the origin represents an astigmatism-free eye. The spread observed in the presurgical data becomes a concentrated data set around the origin after lens implantation. Note that the central open circle located at $(0,0)$ represents 23 eyes that ended up having $0 \mathrm{D}$ of postoperative astigmatism.

3.3. Intraocular Pressure, Endothelial Cell Density, and Vault. Mean IOP at 1 year after surgery was $15.27 \pm 3.03 \mathrm{mmHg}$ (range: 9 to $20 \mathrm{mmHg}, p=0.12$ pre vs. post). Figure 3(a) shows the postoperative change in IOP after ICL implantation. It is worth pointing out that no increase exceeding $4 \mathrm{mmHg}$ occurred in any case during the follow-up and that more than $50 \%$ of the eyes experienced a reduction in IOP $(51.62 \%)$. As for the ECD, mean ECD at 1 year was $2516 \pm 234$ cells $/ \mathrm{mm}^{2}$; the change relative to preoperative values did not turn out to be statistically significant (Table $1, p=0.29$ pre vs. post). More specifically, mean change in ECD ((preoperative ECD-postoperative ECD) $\times 100 /$ preoperative ECD) was $2.04 \%$. Lastly, mean postoperative vault was $320 \pm 136 \mu \mathrm{m}$ at 1 year (range: 96 to $603 \mu \mathrm{m}$ ). Figure 3(b) shows the postoperative distribution of these vault values. The most prevalent vault range was from 201 to $300 \mu \mathrm{m}$ (9 eyes; $31.03 \%$ ) followed by the 301 to $400 \mu \mathrm{m}$ range (7 eyes; $24.14 \%)$. No eyes showed a vault larger than $701 \mu \mathrm{m}$.

3.4. Adverse Events. There were not any complications (neither intraoperative nor postoperative ones); in fact, all ICL implantations were uneventful. Over 1 year of follow- 


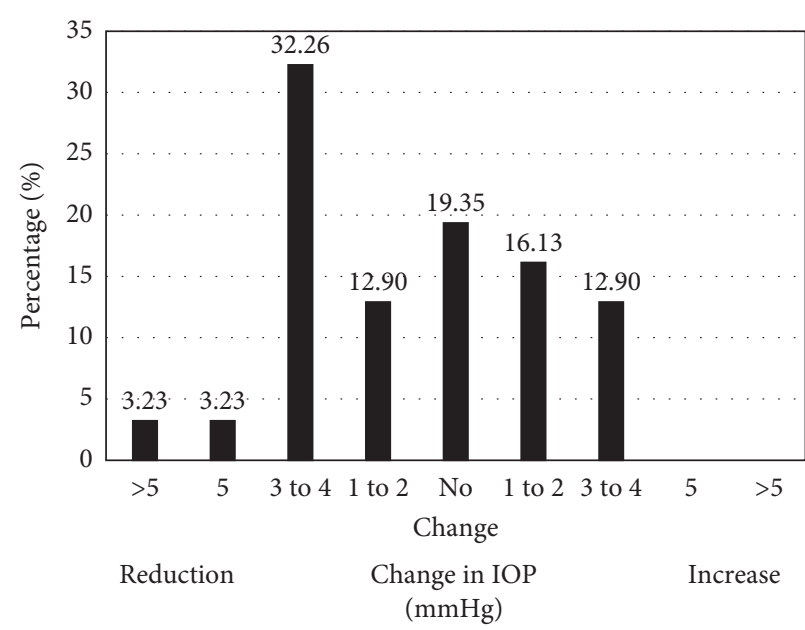

(a)

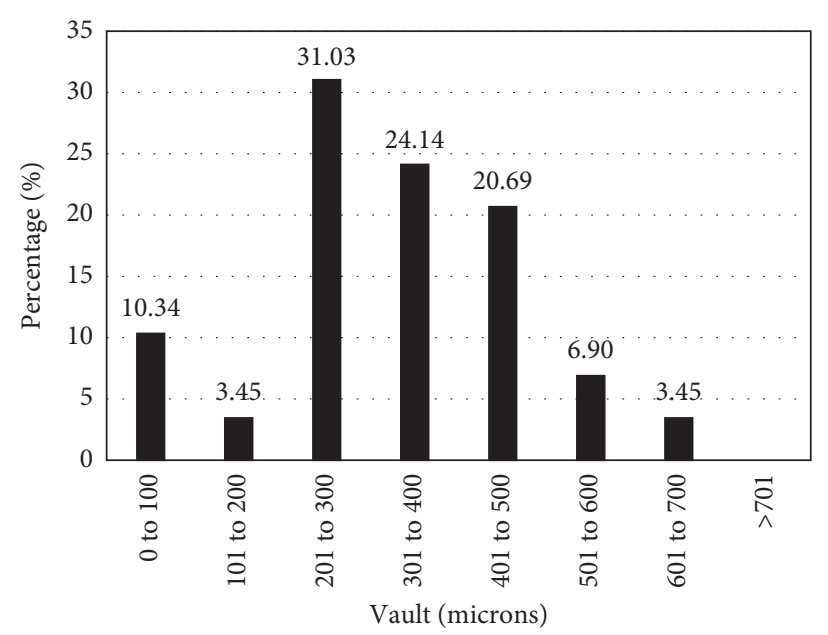

(b)

FIgURe 3: Postoperative changes in intraocular pressure (IOP), measured in mmHg, at 1 year after surgery (a), and distribution of eyes according to the vault, measured in microns, at 1 year after surgery (b).

up, no cases of anterior subcapsular opacity, cataract, pigment dispersion glaucoma, pupillary block, or other visionthreatening complications were reported.

\section{Discussion}

In the present study, we have assessed the visual and refractive 1-year postoperative outcomes yielded by the V4c ICL model in a group of patients aged over 40 . The objective of the present study was to find out whether this model is a safe, effective, and predictable option to correct myopia and myopic astigmatism in this cohort of patients.

The visual outcomes found in our series were satisfactory in terms of safety and efficacy indexes. Namely, the safety index was 1.09, and none of the eyes had lost any line of CDVA, whereas $42.42 \%$ of them gained 1 line or more of CDVA (Figure 1(c)). Most of the eyes maintained their CDVA (57.58\%). The percentage of eyes with 20/20 CDVA increased from $54.55 \%$ preoperatively to more than $69.70 \%$ postoperatively (Figure 2(b)). Similarly, there was also an increase for the 20/25 category, namely, from $72.73 \%$ preoperatively to $90.91 \%$ after surgery. As for the efficacy index, it was 1 . Note also that most eyes had an UDVA of 20/25 or better ( $>75 \%$, Figure 1(a)). Like the one we have introduced, there are different studies in the literature analysing this lens with short, medium, and long follow-up periods $[1,2]$. In order to compare our outcomes with those published previously, we have considered for the discussion only those studies reporting data for the V4c ICL model at 1 year of follow-up. To do that, a literature review search was carried out including the following databases: PubMed (US National Library of Medicine), Web of Science (Thomson Reuters), and Scopus (Elsevier, BV). The search was limited to publications written in English. The date of the last electronic search was March 24, 2020. The literature search based on these criteria resulted in 8 relevant contributions being considered [5-12]. Table 2 summarizes the information about the sample size (number of eyes), patients' age, and SE values of these studies. In addition to this, Table 3 shows these studies' visual outcomes. For example, our safety and efficacy indexes were similar to those reported by Lisa et al. [5], 1.04 and 1.0, respectively, and slightly different from other authors'. Note that in Lisa et al.'s study [5] their preoperative SE was similar to our sample's (about $-8.50 \mathrm{D}$ ). However, we want to point out that, in addition to possible differences across studies in terms of preoperative SE values, mean age and age range also varied significantly across studies (see Table 2). For instance, mean age and SE in Sachdev et al.'s [12] study were 24 years and -9.44 D, whereas in Kamiya et al.'s [9], for the low-to-moderate myopia group, they were 34.8 years and $-4.29 \mathrm{D}$, respectively.

If we focus now on predictability, we can consider these procedures to be successful, since $90.6 \%$ and $93.9 \%$ of the eyes were within the \pm 0.50 and $\pm 1.00 \mathrm{D}$ range of postoperative refractive error, respectively. Both values were also comparable and sometimes better (versus Ganesh et al. [7], García-de-la-Rosa et al. [10], and Niu et al. [11]) than those found in previous studies (see Table 3). Similarly, SE was also good, being below a quarter of diopter $(-0.09 \pm 0.47 \mathrm{D})$. From the same figure, we may observe that 22 eyes $(66.7 \%)$ were within $\pm 0.13 \mathrm{D}(A)$ and a high concentration of points around the origin of the graph $(0,0)$ corresponding to a value of $0 \mathrm{D}$ of astigmatism (C). The patients included in our sample were about 10 years older than those in Kamiya et al.'s sample [9] (this was the study with the highest mean age), whereas mean SE and percentage of eyes \pm 0.50 and $\pm 1.00 \mathrm{D}$ were comparable. Taking into account our outcomes and those previously published with different samples, our results are similar and showed the excellent predictability of the procedure in these patients. The age of the patient does not seem to be a factor that has an impact upon the procedure's predictability. 
TABLE 2: Studies reporting data for the V4c ICL with 1 year of follow-up: year of publication, sample age, and preoperative spherical equivalent (SE) (mean, standard deviation, and range).

\begin{tabular}{lcccc}
\hline Author & Year & Eyes (patients) & Age (ys) & SE (D) \\
\hline Lisa et al. [5] & 2015 & $147(80)$ & $30.4 \pm 4.8(20$ to 40$)$ & $-8.80 \pm 2.60(-2.75$ to -17.50 sph 0 to -3.00 cyl) \\
Karandikar et al. [6] & 2015 & $34(34)$ & $26.13 \pm 3.8(\mathrm{NR})$ & $-9.24 \pm 2.4$ (NR) \\
Ganesh et al. [7] & 2017 & NR (NR) & $26.4 \pm 2.4(\mathrm{NR})$ & $-5.98 \pm 1.15$ (NR) \\
Pjano et al. [8] & 2017 & $28(16)$ & $28.21 \pm 4.06(21$ to 35) & $-9.52 \pm 3.69$ (NR) \\
Kamiya et al. [9] & 2018 & & & $-4.29 \pm 1.31(-0.50$ to -5.88$)$ \\
$\quad$ Low-to-moderate myopia & & $57(57)$ & $34.8 \pm 7.4(20$ to 57) & $-10.13 \pm 2.64(-6.00$ to -18.63$)$ \\
$\quad$ High myopia & & $294(294)$ & $33.6 \pm 7.3(18$ to 54) & $-11.94 \pm 3.51(-7.50$ to -22.88$)$ \\
García-de la Rosa et al. [10] & 2018 & $76(42)$ & $27.4 \pm 5.14(20$ to 39) & $-14.03 \pm 4.46(-7.50$ to -25.75$)$ \\
Niu et al. [11] & 2019 & $40(31)$ & $32.45 \pm 6.85(20$ to 42$)$ & $-9.44 \pm 4.20(-1.75$ to -21$)$ \\
Sachdev et al. [12] & 2020 & $203(\mathrm{NR})$ & $24 \pm \mathrm{NR}(22$ to 28) & $-8.46 \pm 3.31(-1.88$ to -14.88$)$ \\
Current study & 2020 & $33(21)$ & $43.52 \pm 4.49(40$ to 56) & \\
\hline
\end{tabular}

TABLE 3: Visual and refractive outcomes, intraocular pressure (IOP), endothelial cell density (ECD), and vault of the different studies considered (mean, standard deviation, and range) NR: not reported.

\begin{tabular}{|c|c|c|c|c|c|c|c|c|c|c|}
\hline Author & $\begin{array}{c}\text { UCVA } \\
\text { (logMAR) }\end{array}$ & $\begin{array}{c}\text { CDVA } \\
\text { (logMAR) }\end{array}$ & $\begin{array}{l}\text { Safety } \\
\text { index }\end{array}$ & $\begin{array}{l}\text { Efficacy } \\
\text { index }\end{array}$ & $\begin{array}{l} \pm 0.50 \mathrm{D} \\
(\% \text { eyes })\end{array}$ & $\begin{array}{c} \pm 1.00 \mathrm{D} \\
\text { (\% eyes) }\end{array}$ & SE (D) & $\begin{array}{c}\text { IOP } \\
(\mathrm{mmHg}) \\
(\text { eyes }>21)\end{array}$ & $\begin{array}{c}\text { ECD } \\
(\text { cells/m²) } \\
(\% \text { loss })\end{array}$ & Vault $(\mu \mathrm{m})$ \\
\hline Lisa et al. [5] & $0.028 \pm 0.055$ & $0.003 \pm 0.013$ & 1.04 & 1.00 & 93.9 & 100 & $-0.14 \pm 0.26$ & $\begin{array}{c}12.4 \pm 1.4 \\
(0>20)\end{array}$ & $\begin{array}{c}2650 \pm 438 \\
(1.7)\end{array}$ & $\begin{array}{l}405.5 \pm 184.7 \\
(100 \text { to } 980)\end{array}$ \\
\hline $\begin{array}{l}\text { Karandikar } \\
\text { et al. [6] }\end{array}$ & NR & NR & 1.15 & 1.6 & 57.12 & 98.12 & $-0.19 \pm 1.18$ & $19.1 \pm 1.3$ & NR (7.1) & $\begin{array}{l}628.2 \pm 300.1 \\
(\mathrm{NR})\end{array}$ \\
\hline $\begin{array}{l}\text { Ganesh et al. } \\
\text { [7] }\end{array}$ & $-0.022 \pm 0.021$ & $-0.071 \pm 0.079$ & 1.24 & 1.12 & 90 & 100 & $-0.164 \pm 0.20$ & NR & $\begin{array}{c}2808 \pm 315 \\
\quad(9.0)\end{array}$ & NR \\
\hline $\begin{array}{l}\text { Pjano et al. } \\
\text { [8] } \\
\text { Kamiya et al. } \\
{[9]}\end{array}$ & $\begin{array}{c}0.76 \pm 0.16 \\
\text { (decimal) }\end{array}$ & $\begin{array}{c}0.79 \pm 0.14 \\
(\text { decimal })\end{array}$ & 1.25 & 1.2 & NR & NR & $-0.21 \pm 0.27$ & $14.96 \pm 1.7$ & $\begin{array}{c}2512 \pm 127 \\
(5.5)\end{array}$ & NR \\
\hline $\begin{array}{l}\text { Low-to- } \\
\text { moderate } \\
\text { myopia }\end{array}$ & $-0.17 \pm 0.14$ & $-0.21 \pm 0.10$ & NR & NR & 93 & 98 & -0.12 & $13.1(0)$ & NR $(0.1)$ & NR \\
\hline $\begin{array}{l}\text { High } \\
\text { myopia }\end{array}$ & $-0.16 \pm 0.09$ & $-0.21 \pm 0.08$ & NR & NR & 94 & 99 & 0.02 & $13.6(0)$ & NR $(0.1)$ & NR \\
\hline $\begin{array}{l}\text { García-de la } \\
\text { Rosa et al. } \\
{[10]}\end{array}$ & $0.12 \pm 0.12$ & $0.05 \pm 0.08$ & NR & NR & 85 & 86 & $-0.06 \pm 0.77$ & NR & NR & $\begin{array}{l}449 \pm 180 \\
(\mathrm{NR})\end{array}$ \\
\hline $\begin{array}{l}\text { Niu et al. } \\
\text { [11] }\end{array}$ & $\begin{array}{c}0.89 \pm 0.30 \\
(\text { decimal })\end{array}$ & $\begin{array}{c}1.00 \pm 0.27 \\
(\text { decimal })\end{array}$ & 1.33 & 1.14 & 69 & 92 & $-0.67 \pm 1.29$ & $\begin{array}{c}15.15 \pm 2.57 \\
(0)\end{array}$ & $\begin{array}{c}2963 \pm 396 \\
(8.38)\end{array}$ & $\begin{array}{c}380 \pm 152 \\
(90 \text { to } 700)\end{array}$ \\
\hline $\begin{array}{l}\text { Sachdev } \\
\text { et al. [12] }\end{array}$ & $0 \pm \mathrm{NR}$ & $0 \pm N R$ & NR & NR & 94.09 & 96.09 & $-0.12 \pm \mathrm{NR}$ & NR & NR & NR \\
\hline $\begin{array}{l}\text { Current } \\
\text { study }\end{array}$ & $\begin{array}{c}0.88 \pm 0.16 \\
(\text { decimal })\end{array}$ & $\begin{array}{c}0.96 \pm 0.09 \\
(\text { decimal })\end{array}$ & 1.09 & 1.00 & 90.6 & 93.9 & $-0.09 \pm 0.47$ & $\begin{array}{c}15.27 \pm 3.03 \\
(0)\end{array}$ & $\begin{array}{c}2516 \pm 234 \\
(2.04)\end{array}$ & $\begin{array}{c}320 \pm 136 \\
(96 \text { to } 603)\end{array}$ \\
\hline
\end{tabular}

UCVA: uncorrected distance visual acuity, CDVA: corrected distance visual acuity, SE: spherical equivalent, and NR: not reported.

Some surgeons may consider implanting this lens following a monovision or undercorrection strategy. This approach was attempted by Kamiya et al. [3] and Takahashi et al. [3]. The first study assessed 34 eyes, mean patients' age was 46.1 years (from 40 to 53 years), and mean SE was -8.67 $\mathrm{D}$ (from -2.25 to $-18.25 \mathrm{D}$ ); the second study included 42 eyes, mean patients' age was 45 years (from 40 to 53 years), and mean SE was $-7.37 \mathrm{D}$ (from -2.25 to $-14.75 \mathrm{D}$ ). Both studies reported their outcomes at 6 months after surgery. The first study selected emmetropia in the dominant eye and -0.5 to $-1.0 \mathrm{D}$ in the nondominant eye as their target refraction, whereas the second study approximately -0.50 to $-1.50 \mathrm{D}$ in both eyes. They reported that $100 \%$ of eyes were within the $\pm 0.50 \mathrm{D}$ range and the mean UDVA and CDVA were about -0.04 and $-0.19 \log$ MAR, respectively. Both studies concluded that monovision [3] or intentional undercorrection [4] provided good binocular vision at near to far distances, suggesting its feasibility as a new surgical presbyopic approach for early presbyopia. We have not considered this option in our series.

Regarding IOP, in our study mean IOP was $15.27 \pm 3.03 \mathrm{mmHg}$ (range: 9 to $20 \mathrm{mmHg}$ ), which is similar to that found before surgery $(p=0.12)$. Figure $3(\mathrm{a})$ shows the postoperative change in IOP after ICL implantation, and from this figure we may observe that $51.62 \%$ of eyes showed a reduction, $19.35 \%$ of eyes no change, and $29.03 \%$ of eyes a mild increase in IOP compared to preoperative values. No acute postoperative hypertension and no increase of more 
than $4 \mathrm{mmHg}$ occurred in any case during the follow-up. Our results (mean IOP $=15.27 \pm 3.03 \mathrm{mmHg}$ ) are in good agreement with those found by other authors at 1 year (about $15 \mathrm{mmHg}$, see Table 3). The central port of the lens, designed to allow physiologic flow of aqueous without resorting to $\mathrm{Nd}$ :YAG peripheral iridotomies or intraoperative iridectomies, confirms that IOP was well controlled after surgery. However, our follow-up of 1 year is short and these patients should be kept under follow-up for longer periods. Shimizu et al. [13] and Alfonso et al. [14] with 5 years of follow-up in younger patients implanted with this lens followed this approach of extended follow-up period (mean ages of 31.9 and 31.2 years, respectively). Our mean ECD at 1 year showed no statistically significant differences with their corresponding preoperative values $\left(2516 \pm 234\right.$ vs. $2568 \pm 332$ cells $\left./ \mathrm{mm}^{2}, p=0.29\right)$. These mean values were similar to those found in previous studies, which ranged from 2512 [8] to 2963 [11] cells $/ \mathrm{mm}^{2}$. The percentage loss in our sample amounted to $2.04 \%$, whereas in previous studies it was rather diverse, ranging from $1.7 \%$ [5] to $9.0 \%$ [7]. A physiologic cell loss of approximately $0.6 \%$ per year $[15,16]$ is to be expected, although higher values of loss may happen at early times related to the surgical procedure.

As for vault, we have found a mean vault of $320 \mu \mathrm{m}$ in our patients. Figure 3(b) shows the exact distribution of vault per interval, the $201-300 \mu \mathrm{m}$ interval being the range corresponding to the highest percentage of eyes $(31.03 \%)$. Few studies in the literature reported vault values (see Table 3); however, those studies that measured it showed values ranging from 380 [11] to $628 \mu \mathrm{m}$ [6], which are slightly higher than what we found in our study; this could be connected with the changes that happen in the crystalline lens with aging. Vault is negatively correlated with age [17]. ACD decreases with age because of the thickening of the aging crystalline lens [18], which results in a lower vault. Such decrease in ACD is likely to be induced by the thickening of the aging crystalline lens, which happens at an average rate of $24 \mu \mathrm{m} /$ year [19]. A review of potential complications of ICL implantation concluded that low vault is related to cataract formation [20] and the rate of vault reduction is faster in patients who developed lens opacity $[21,22]$. The change in vault with time has been also reported in a long follow-up study with this specific model. The 5-year study carried out by Alfonso et al. [14] showed a reduction of about $1.2 \mu \mathrm{m} / \mathrm{month}$. Changes with time (due to crystalline lens thickening) should be always borne in mind, especially in patients with low vault values.

No postoperative adverse events occurred during the 1 year of follow-up. Previous studies have described some postoperative complications that include ECD loss, IOP increase, or cataracts [20]. It seems that cataract formation is the most frequent complication after ICL implantation; this may be linked to the patient's age at the time of surgery. Lackner et al. [23] indicated that the most important factor that influences the likelihood of crystalline lens opacification was the age of the patient, and potentially, its vulnerability and susceptibility increase with advanced age. They reported in a series of 76 eyes that all occurrences of late cataract development $(n=11)$ were in patients older than 50 years of age. Similarly, Gonvers et al. [24] found higher incidence of cataract development in older patients ( $14 \%$ of the younger patients [10 to 40 years] versus $37 \%$ of the older patients [ 41 to 50 years]). Choi et al. [25], in a 10-year clinical study $(n=110)$, found lens opacities in 21 eyes $(12.1 \%)$ and the mean age at which lens opacity was first observed was $48.7 \pm 7.1$ years. They found a difference in the preoperative age between the clear lens group and lens opacity group as well as a higher cumulative incidence of lens opacity in patients with higher preoperative age. In addition, their mean vault value was significantly lower in older patients 10 years after ICL implantation, whereas it did not differ significantly at 6 months. In the same way, Lindland et al. [26] also reported lower vault in older patients over a follow-up of about 5 years. However, all these studies evaluated patients implanted with older ICL models. With the present V4c ICL, recent long-term studies $[13,14]$ and meta-analysis reported no postoperative complications [1,2]. Kamiya et al. [3] and Takahashi et al. [4], in an older patient sample (with 6 months of follow-up) similar to ours, did not report either asymptomatic or symptomatic cataract formation. Thus, it seems that a new lens design (which improves the circulation of the aqueous humor to the anterior surface of the crystalline lens [13]) and new anterior-segment imaging systems being used (providing accurate measurements of several parameters mandatory for ICL size calculation) are essential factors that may contribute to the zero-incidence of cataract formation. With accurate vault measurement and controlled follow-up, the risk for lens opacity in patients who have ICL implantation over 40 years seems to be minimal. However, we need to bear in mind that our study's sample is small and that the follow-up was short. A large cohort of patients with longer follow-up periods is necessary to confirm our outcomes. Some limitations of our study are, in addition to the small sample and short follow-up, the lack of measurement of near vision and crystalline lens rise, which should be included in future studies.

In conclusion, the V4c ICL implanted in patients over 40 years showed good visual outcomes, resulting in improved UDVA and CDVA after surgery. The refractive outcomes were also successful, confirming the procedure's high predictability. Our results are in good agreement with previous studies performed in younger samples. So, this lens may be considered a good procedure to correct myopia and myopic astigmatism in older patients. Future studies with larger samples and longer follow-up periods are needed to fully characterize this lens' outcomes in this specific patients' age range.

\section{Data Availability}

All data generated or analysed during this study are available within the article.

\section{Conflicts of Interest}

Robert Montes-Micó declares research and consulting contracts with Alcon Laboratories, Essilor, Glaukos, and STAAR Surgical through the University of Valencia. 


\section{References}

[1] M. Packer, "The Implantable Collamer Lens with a central port: review of the literature," Clinical Ophthalmology, vol. 12, pp. 2427-2438, 2018.

[2] Y. Tang and J. Ye, "Phakic posterior chamber intraocular lens with a central hole in treating patients with moderate to high myopia: a meta-analysis," Journal of Ophthalmology, 2019.

[3] K. Kamiya, M. Takahashi, N. Takahashi, N. Shoji, and K. Shimizu, "Monovision by implantation of posterior chamber phakic intraocular lens with a central hole (hole ICL) for early presbyopia," Science Reports, vol. 7, p. 11302, 2017.

[4] M. Takahashi, K. Kamiya, N. Shoji, S. Kato, A. Igarashi, and K. Shimizu, "Intentional undercorrection by implantation of posterior chamber phakic intraocular lens with a central hole (hole ICL) for early presbyopia," BioMed Research International, vol. 10, Article ID 6158520, , 2018.

[5] C. Lisa, M. Naveiras, B. Alfonso-Bartolozzi, L. Belda-Salmerón, R. Montés-Micó, and J. F. Alfonso, "Posterior chamber collagen copolymer phakic intraocular lens with a central hole to correct myopia: one-year follow-up," Journal of Cataract \& Refractive Surgery, vol. 41, no. 6, pp. 1153-1159, 2015.

[6] S. Karandikar, V. Bhandari, and J. Reddy, "Outcomes of implantable collamer lens V4 and V4c for correction of high myopia-a case series," Nepalese Journal of Ophthalmology, vol. 7, pp. 164-172, 2105.

[7] S. Ganesh, S. Brar, and A. Pawar, "Matched population comparison of visual outcomes and patient satisfaction between 3 modalities for the correction of low to moderate myopic astigmatism," Clinical Ophthalmology, vol. 11, pp. 1253-1263, 2017.

[8] M. Pjano, A. Pidro, A. Biscevic, S. Grisevi, B. Pandzi, and V. Cerovic, "Refractive outcomes of posterior chamber phakic intraocular lens implantation for correction of myopia and myopic astigmatism," Medical Archives, vol. 71, no. 2, pp. 93-96, 2017.

[9] K. Kamiya, K. Shimizu, A. Igarashi et al., "Posterior chamber phakic intraocular lens implantation: comparative, multicentre study in 351 eyes with low-to-moderate or high myopia," British Journal of Ophthalmology, vol. 102, no. 2, pp. 177-181, 2018.

[10] G. Garcia-De la Rosa, A. Olivo-Payne, J. C. Serna-Ojeda et al., "Anterior segment optical coherence tomography angle and vault analysis after toric and non-toric implantable collamer lens V4c implantation in patients with high myopia," British Journal of Ophthalmology, vol. 102, no. 4, pp. 544-548, 2018.

[11] L. Niu, H. Miao, T. Han, L. Ding, X. Wang, and X. Zhou, "Visual outcomes of visian ICL implantation for high myopia in patients with shallow anterior chamber depth," BMC Ophthalmology, vol. 19, p. 121, 2019.

[12] G. Sachdev, S. Singh, S. Ramamurthy, N. Rajpal, and R. Dandapani, "Comparative analysis of clinical outcomes between two types of posterior chamber phakic intraocular lenses for correction of myopia and myopic astigmatism," Indian Journal of Ophthalmology, vol. 67, no. 7, pp. 1061-1065, 2019.

[13] K. Shimizu, K. Kamiya, A. Igarashi, and H. Kobashi, "Longterm comparison of posterior chamber phakic intraocular lens with and without a central hole (hole ICL and conventional ICL) implantation for moderate to high myopia and myopic astigmatism: consort-compliant article," Medicine (Baltimore), vol. 95, Article ID e3270, 2016.
[14] J. F. Alfonso, L. Fernández-Vega-Cueto, B. Alfonso-Bartolozzi, R. Montés-Micó, and L. Fernández-Vega, "Five-year follow-up of correction of myopia: posterior chamber phakic intraocular lens with a central port design," Journal of Refractive Surgery, vol. 35, no. 3, pp. 169-176, 2019.

[15] H. F. Edelhauser, D. R. Sanders, R. Azar, H. Lamielle, and ICL in Treatment of Myopia Study Group, "Corneal endothelial assessment after ICL implantation," Journal of Cataract \& Refractive Surgery, vol. 30, no. 3, pp. 576-583, 2004.

[16] W. M. Bourne, L. R. Nelson, and D. O. Hodge, "Central corneal endothelial cell changes over a ten-year period," Investigative Ophthalmology \&amp; Visual Science, vol. 38, no. 3, pp. 779-782, 1997.

[17] J. F. Alfonso, L. Fernández-Vega, C. Lisa, P. Fernandes, J. Jorge, and R. M. Micó, "Central vault after phakic intraocular lens implantation: correlation with anterior chamber depth, white-to-white distance, spherical equivalent, and patient age," Journal of Cataract \& Refractive Surgery, vol. 38, no. 1, pp. 46-53, 2012.

[18] M. Dubbelman, G. L. van der Heijde, H. A. Weeber, and G. F. J. M. Vrensen, "Changes in the internal structure of the human crystalline lens with age and accommodation," Vision Research, vol. 43, no. 22, pp. 2363-2375, 2003.

[19] D. A. Atchison, E. L. Markwell, S. Kasthurirangan, J. M. Pope, G. Smith, and P. G. Swann, "Age-related changes in optical and biometric characteristics of emmetropic eyes," J Vis, vol. 29, pp. 1-20, 2008.

[20] P. Fernandes, J. M. González-Méijome, D. Madrid-Costa, T. Ferrer-Blasco, J. Jorge, and R. Montés-Micó, "Implantable collamer posterior chamber intraocular lenses: a review of potential complications," Journal of Refractive Surgery, vol. 27, no. 10, pp. 765-776, 2011.

[21] G. Schmidinger, B. Lackner, S. Pieh, and C. Skorpik, "Longterm changes in posterior chamber phakic intraocular collamer lens vaulting in myopic patients," Ophthalmology, vol. 117, no. 8, pp. 1506-1511, 2010.

[22] I. Guber, V. Mouvet, C. Bergin, S. Perritaz, P. Othenin-Girard, and F. Majo, "Clinical outcomes and cataract formation rates in eyes 10 years after posterior phakic lens implantation for myopia," JAMA Ophthalmology, vol. 134, no. 5, pp. 487-494, 2016.

[23] B. Lackner, S. Pieh, G. Schmidinger et al., "Long-term results of implantation of phakic posterior chamber intraocular lenses," Journal of Cataract \& Refractive Surgery, vol. 30, no. 11, pp. 2269-2276, 2004.

[24] M. Gonvers, C. Bornet, and P. Othenin-Girard, "Implantable contact lens for moderate to high myopia," Journal of Cataract \& Refractive Surgery, vol. 29, no. 5, pp. 918-924, 2003.

[25] J. H. Choi, D. H. Lim, S. W. Nam, C. M. Yang, E. S. Chung, and T.-Y. Chung, "Ten-year clinical outcomes after implantation of a posterior chamber phakic intraocular lens for myopia," Journal of Cataract \& Refractive Surgery, vol. 45, no. 11, pp. 1555-1561, 2019.

[26] A. Lindland, H. Heger, M. Kugelberg, and C. Zetterström, "Vaulting of myopic and toric implantable collamer lenses during accommodation measured with Visante optical coherence tomography," Ophthalmology, vol. 117, no. 6, pp. 1245-1250, 2010. 